\title{
Neural Circuits Mediating Visual Flight Control in Flies. II. Separation of Two Control Systems by Microsurgical Brain Lesions
}

\author{
Klaus Hausen ${ }^{a}$ and Christian Wehrhahn \\ Max-Planck-Institut für biologische Kybernetik, D-7400 Tübingen, Federal Republic of Germany
}

The role of 2 sets of interneurons in the optic lobes of blowflies in visual course control was studied by means of brain lesions. The first set comprises the cells $\mathrm{HS}$ and $\mathrm{H} 2$, which respond to global horizontal motion. The second set are the FD-cells, which respond selectively to local horizontal motion. All these cells are output neurons of the third optic ganglion of flies and are thought to be coupled via descending neurons to the flight motor system (Fig. 1, a-c).

In 2 series of experiments specific cells of these 2 sets were inactivated by microsurgical brain lesions $L 1$ and L2 respectively (Fig. 1c). The effects of the lesions on visual course control were tested by measuring the yaw torque responses of the animals in restrained flight before and after the operation. The flies were stimulated in these tests with monocular and binocular motion of periodic gratings moving in either the horizontal or the vertical direction.

Lesion L 1 in the right side of the brain inactivates the right HS-cells and the left H2- and FD-cells. This leads to a complete block of the response to binocular clockwise horizontal motion and a reduction of the response to monocular motion from front to back on the right side of the animal. Application of $L 1$ also leads to a pronounced response to binocular motion from front to back not observed in normal animals (Fig. 2). The response to monocular vertical motion is unaffected (Fig. 4). Lesion L2 reduces all responses to monocular and binocular horizontal motion present in normal animals (Fig. 2).

The behavioral effects of the lesions are highly specific and consistent with predictions based on the well-known anatomical and physiological properties of the neural circuitry investigated. The results demonstrate directly that the HS-, H2-, and FD-cells control motion-induced steering maneuvers in flight.

Recent studies on visual orientation in flies indicate that course control during flight is mediated by neural circuits that compute global and local retinal motion patterns (reviews: Reichardt, 1986; Egelhaaf et al., 1988; Hausen and Egelhaaf, 1989). Car-

\footnotetext{
Received May 31, 1989; revised July 21, 1989; accepted July 24, 1989.

T. Wiegand and $\mathbf{H}$. Kümmerle provided expert technical assistance in this tough project. T. Wiegand also prepared the figures. W. Reichardt and M. Egelhaaf contributed numerous discussions and comments on the manuscript. The comments of one of the 2 anonymous referees made the manuscript substantially more comprehensible. I. Geiss edited the manuscript. We thank them all.

Correspondence should be addressed to Christian Wehrhahn, Max-Planck-Institut für biologische Kybernetik, Spemannstrasse 38, D-7400 Tübingen, Federal Republic of Germany.

a Present address: Zoologisches Institut der Universität Köln, Weyertal 119 , $5000 \mathrm{Köln} \mathrm{41,} \mathrm{Federal} \mathrm{Republic} \mathrm{of} \mathrm{Germany.}$

Copyright (C) 1990 Society for Neuroscience $0270-6474 / 90 / 010351-10 \$ 02.00 / 0$
}

dinal interneurons of these circuits are the horizontal cells (HScells; Hausen, 1982a, b), which signal course deviations due to self-rotations of the animal, and the figure detection cells (FDcells; Egelhaaf, 1985b, c), which signal horizontal object motion. Both types of cells are thought to control yaw torque generation by the flight motor during steering maneuvers. Since the neural connections between the HS- and the FD-cells and the motor system are not yet fully analyzed (but see Egelhaaf, 1989), the role of these cells in visual course control could only be inferred from comparison of electrophysiological and behavioral data.

In order to relate behavioral and neuronal findings, the first part of this study (Hausen and Wehrhahn, 1989) investigated the responses of the HS-cells and the yaw torque responses of tethered flying blowflies (Calliphora) under identical stimulus conditions. The results of these experiments yielded clear evidence in favor of the proposed role of the cells in course control. It was further demonstrated that the response behavior of $\mathrm{Cal}$ liphora and the housefly Musca under these experimental conditions is highly similar. This suggests analogous neural course control circuits in both species.

In the present account, the concept of course control by HSand FD-cells is tested directly by lesioning the axonal pathways of the cells in the brain of Calliphora and evaluating the resulting behavioral effects.

In order to clarify the rationale of these experiments, the neural circuitry investigated will be first described in some detail.

\section{Cellular organization of the two control circuits}

Tethered flies generate yaw torque responses when stimulated with binocular horizontal rotatory motion as sketched in Figure la (Götz, 1968; Wehrhahn, 1986). A simplified scheme of the neural circuits assumed to mediate these responses is outlined in Figure $1 b$. The horizontal system (HS) in each optic lobe responds selectively to ipsilateral front-to-back and contralateral back-to-front motion. It activates the steering muscles of the flight motor such that the flight force of the ipsilateral wing is decreased whereas that of the contralateral wing is increased. This differential modulation of the flight forces leads to generation of yaw torque turning the flying animal into the same direction as the perceived motion ("classical" optomotor response). The second circuit contains the FD-cells, which are activated by local horizontal motion in either direction and modulate the flight force generation in the same way as the horizontal system. Monocular stimulation of the FD-system thus leads to turning of the animal toward the moving stimulus irrespective of its direction of motion (fixation response).

The actual synaptic connection of both circuits is complex (Fig. 1c). Both the HS- and FD-cells are large-output cells of 

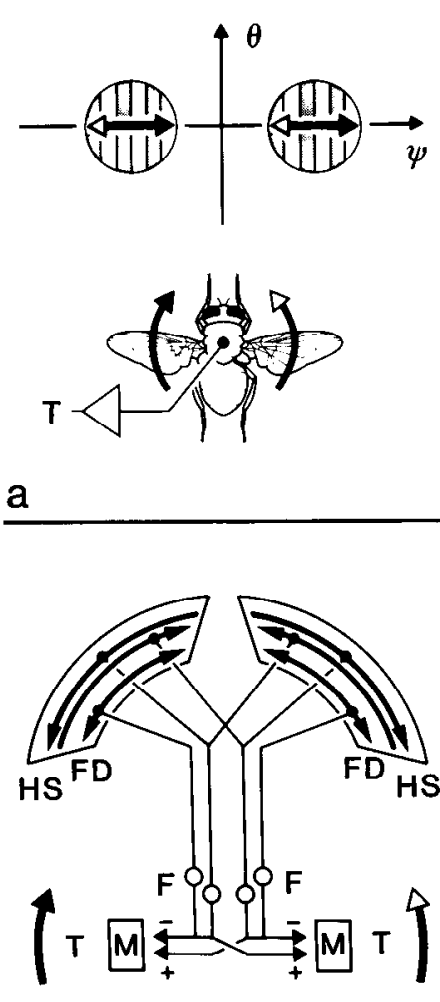

b
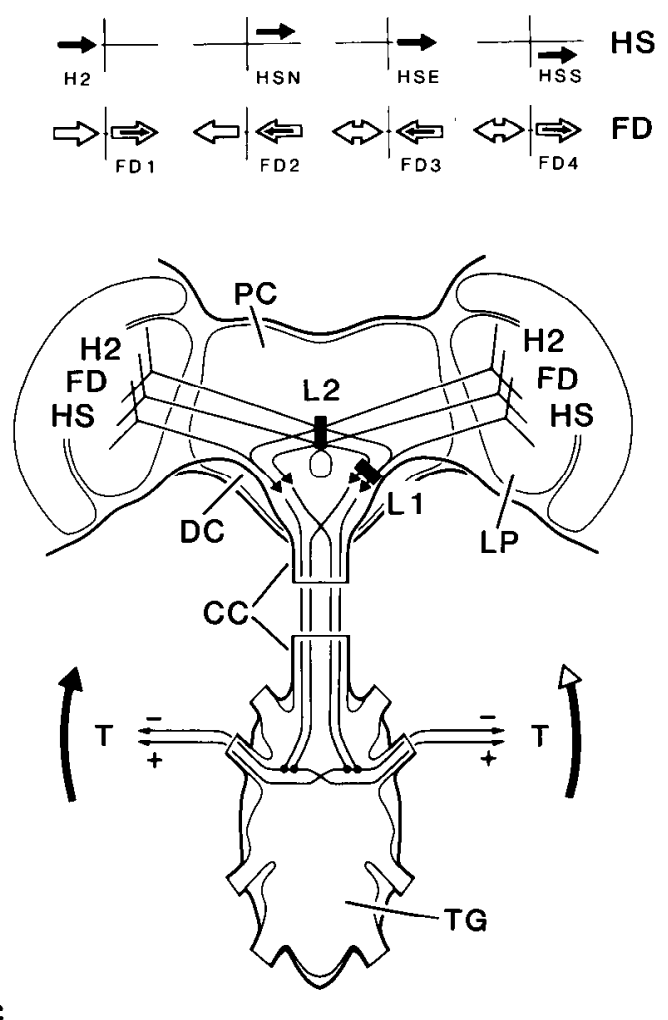

Figure 1. The concept of neural yaw torque control during flight in flies. a, Experimental setup for yaw torque measurements in tethered flight. The animal is stimulated with moving periodic gratings presented in the frontolateral visual field at $\theta=0^{\circ}, \psi=40^{\circ}$, as illustrated. The flying animal is attached to a torque meter, which allows registration of the motion-induced yaw torque responses $(T)$. Stimulation with clockwise and counterclockwise motion leads to torque responses following the direction of motion. $b$, Schema of the proposed yaw torque control system. Horizontal motion is evaluated by 2 neural systems behind each eye. Horizontal cells $(H S)$ are activated by global ipsilateral front-to-back and contralateral back-to-front motion. Figure detection cells $(F D)$ respond selectively to local horizontal motion in the ipsilateral visual field. Both systems induce differential modulation of the flight forces produced by the ipsilateral and contralateral part of the flight motor $(M)$ such that yaw torque $(T)$ is generated. $c$, Cellular wiring of the proposed yaw torque control system in the visual and central nervous system of the fly. The main motion computation center of the visual system is located in the third ganglion of the optic lobe, the lobula plate $(L P)$ (peripheral visual system and compound eyes are not shown). The HS- and FD-cells are directionally selective output neurons of this neuropil projecting into the ipsilateral and contralateral deutocerebrum, respectively. The contralateral axon projections pass through the deep protocerebrum $(P C)$. The HS-cells are postsynaptic to the motion-sensitive H2-cell of the contralateral lobula plate. The HS-cells comprise 3 individual neurons (HSN. HSE. HSS) scanning the dorsal, medial, and ventral ipsilateral visual field. The directional selectivities of the HS-cells of the right lobula plate and the coupled H2-cell of the left lobula plate are indicated by arrows in the $\theta / \psi$ diagram at the top of the figure. Due to the synaptic H2-input, the HS-cells show binocular sensitivity and respond to global rotatory motion. The FD-cells comprise 4 cells $(F D 1-4)$. Their directional selectivities are indicated by thin arrows in the second row of diagrams. Due to directionally selective large-field inhibition (white arrows), FD-cells are selectively activated by local motion (black arrows). Both HS- and FD-cells are assumed to be coupled in the deutocerebrum to descending neurons passing through the cervical connective $(C C)$ into the thoracic ganglion (TG). They are thought to induce differential modulations of the ipsi- and contralateral steering muscles activity leading to torque generation as indicated. The illustrated wiring concept is tested in the present study by disrupting the axonal pathways of FD-, H2-, and HS-cells with microsurgical lesions L1 and L2 and by comparing the motion-induced torque responses of the operated flies with those of normal ties.

the third visual neuropil of the optic lobe, the lobula plate. This neuropil is assumed to receive input from retinotopic arrays of elementary motion detectors, the signals of which are integrated in the dendritic domains of identified interneurons. These have different directional selectivities and encode horizontal and vertical motion in different parts of the ipsilateral visual field (Hausen, 1981, 1984, 1989).

The horizontal system consists of 3 giant neurons termed north, equatorial, and south horizontal cell (HSN, HSE, HSS), which scan the dorsal, equatorial, and ventral part of the ipsilateral visual field, respectively (Hausen 1976, 1982a, b; Eckert, 1981). All 3 cells are excited by horizontal motion from front to back and inhibited by motion from back to front. They respond best to motion of large patterns and show a maximal sensitivity in the frontal part of their receptive fields. The axons of the horizontal cells project directly beneath the posterior surface of the brain into the ipsilateral deutocerebrum, where they give rise to short axon collaterals and terminal arborizations. The collaterals of the HSN and HSE are postsynaptic to the terminal of the $\mathrm{H} 2$-cell of the contralateral lobula plate which responds selectively to motion from back to front. The HSN and HSE must thus be considered binocular elements sensitive to horizontal rotary motion. The directional selectivities of the left H2- and right HS-cells of a fly are indicated by arrows in the top row of diagrams in Figure $1 c$ (thin lines represent the $\theta / \psi$-coordinates shown in Fig. $1 a$ ).

The FD-cells represent a less homogeneous group of lobula plate neurons. At present, 4 cells termed FDI-4 are described 
(Egelhaaf, 1985b). FD1 and FD4 respond selectively to small stimuli moving from front to back. The particular spatial tuning of the cells to local motion is achieved by binocular inhibition under large-field motion in the same direction; the FD4 is additionally inhibited by contralateral motion from front to back. In contrast, FD2 and FD3 are activated by local ipsilateral motion from back to front and are inhibited by ipsilateral largefield motion in this direction. The inhibitory influence of contralateral motion is not yet firmly established in the case of the FD2, whereas inhibition by contralateral horizontal motion in both directions has been demonstrated for the FD3. The second row of diagrams in Figure $1 c$ shows the directional selectivities (black arrows) and the respective inhibitory inputs (white arrows) of the 4 FD-cells. The receptive fields of the FD1 and 2 are located in the frontal visual field of the compound eye, and both cells show maximal sensitivity to motion at about $\psi=10^{\circ}$. The FD3 and FD4 scan the more lateral region of the visual field and show spatial sensitivity maxima at $\psi=40-60^{\circ}$.

At present, the cellular identity of the FD1 is an open question since 2 neurons with different structures but identical functional properties have been described. The first is a heterolateral element the axon of which projects into the contralateral deutocerebrum. The second neuron is a homolateral element terminating in the ipsilateral deutocerebrum. The anatomical identity of the other 3 FD-cells is definite. The FD2 projects into the ipsilateral deutocerebrum, whereas the FD3 and FD4 form, together with the heterolateral FD1, the $\mathrm{H} 2$, and further elements of the lobula plate, a common axonal pathway in the protocerebrum terminating in the area of the contralateral HSterminals. This axonal tract crosses the midline of the brain between the central complex and esophageal channel (Hausen, 1989). The HS-cells, the H2-, and the hetereolateral FD-cells are shown schematically in Figure $1 c$.

Both the HS- and FD-cells are considered to be coupled via the descending neurons to the motor control circuits in the thoracic ganglion. With regard to the HS-cells, direct evidence for a synaptic connection to descending neurons passing down the cervical connective has been gained in anatomical studies (Strausfeld and Bassemir, 1985; Hausen, 1989). In the FD-cells the anatomical demonstration of such synaptic connections is still lacking. Since, however, the whole termination area of the HS- and FD-cells is densely invested by dendrites of descending neurons (Strausfeld and Bacon, 1983), such synaptic contacts are more than likely. The structural organization of the motor control circuits in the thoracic ganglion is presently under investigation. The results available indicate that visual descending neurons, as well as sensory interneurons derived from halteres, wings, and antennae, contact the motoneurons of the steering muscles in the flight motor directly (Hausen and Hengstenberg, 1987; Hausen et al., 1988; Hengstenberg et al., 1988).

Electrophysiological studies have revealed that the steering muscles receive multisensory input and that their activity is modulated by motion in the visual field. It has been further demonstrated that the activity of particular steering muscles and the generation of flight torque are correlated (Heide, 1975, 1983; Spüler, 1980; Hirth, 1981; Götz, 1983; Spüler and Heide, 1987). Thus, although the neural connections between lobula plate and motoneurons are not yet fully unraveled, there is strong evidence for a rather direct transfer of motion information into the flight motor (Egelhaaf, 1989). These connections and their effect on torque generation are also sketched in Figure $1 c$.

In the present experiments this concept is tested by lesioning the output pathways of the lobula plate in 2 areas of the brain and by measuring subsequently the torque responses of the operated animals. By the first lesion ( $\mathrm{Ll}$ in Fig. $1 c$ ) the terminals and thus the output connections of the right HS-cells and the left H2- and FD-cells are disrupted in the deutocerebrum. By the second lesion (L2 in Fig. 1c) the axonal pathways of the H2and FD-cells in the central protoccrcbrum are destroyed. Thus, the torque responses of the lesioned animals to stimulation with motion should reflect the influence of the remaining lobula plate output elements shown in Figure $1 c$.

In the experiments presented below the FD1 and FD2 were only weakly activated by the stimulation device used. We will, therefore, neglect both cells in order to simplify the arguments and will discuss the evidence justifying this omission later.

\section{Materials and Methods}

\section{Animals}

The experiments were performed with female houseflies (Musca domestica) and blowflies (Calliphora erythrocephala) reared in laboratory cultures. The animals were 4-10 d old.

\section{Microsurgical operations}

Flies were immobilized by brief anesthesia with carbon dioxide and were placed dorsal side up between 2 pieces of elastic foam rubber such that their bodies were restrained and the heads remained free. The heads were tilted downward and were temporarily fixed in this position with low-melting-point wax.

Lesion type 1. A small piece of cuticle was removed with a microknife from the rear of the head capsula in order to expose the posterior surface of the right deutocerebrum and the proximal part of the optic lobe containing the lobula plate. A superficial incision was made through the part of the deutocerebrum containing the axons of the horizontal cells of the right lobula plate and the axon terminals of the $\mathrm{H} 2$ - and heterolateral FD-cells of the left lobula plate (the homolateral FD-cells were not affected by this procedure; see lesion L1 in Fig. 1c). Special care was taken to spare the axon bundles of the visual interneurons in the posterior optic tract and the motion-sensitive vertical cells located dorsally of the lesioned area.

Lesion type 2. A flap of cuticle was excised from the central part of the rear head capsula directly above the neck foramen in order to expose the posterior surface of the medial protocerebrum. A deep vertical cut was made through the protocerebrum, which reached from the central complex down to the esophageal channel. This destroyed the axonal pathways of the H2- and FD-cells of both lobula plates, which cross each other below the elipsoid body of the central complex (see lesion L2 in Fig. 1c).

Control operations. In initial control experiments all steps of the described operations were performed except for the actual incisions in the brain.

After the operations the excised pieces of cuticle were replaced in the head capsulas and were fixed with several small drops of wax. Drying hemolymph sealed the margins of the flaps within 10-20 min. The heads of the animals were then moved back into their natural position and fixed to the prothorax by means of a wax bridge. A small cardboard triangle was glued to the scutum, which was used in the subsequent experiments to attach the flies to a torque meter. The flies were finally released from the foam rubber and were put into a flight cage. They were allowed to recover from the operation until the next day.

\section{Behavioral tests}

All animals were tested before and after the operation in behavioral experiments in which their yaw torque responses to visual stimulation with motion were measured.

The stimulation device was identical to that used in the first part of this study and consisted of 2 periodic gratings placed symmetrically in front of the left and right eye at $\theta=0^{\circ}$ and $\psi=40^{\circ}, \theta$ and $\psi$ denoting elevation and azimuth in the visual field as sketched in Figure $1 a$. The gratings consisted of dark and bright stripes (grating diameter: $d=39.6^{\circ}$, spatial wavelength: $\lambda=13.2^{\circ}$, contrast: $c=0.8$, mean luminance: $I=$ $\left.70 \mathrm{~cd} / \mathrm{m}^{2}\right)$ and were moved with a contrast frequency of $1 \mathrm{~Hz}$ according 
to a standard program in which monocular or binocular motion stimuli were repetitively displayed for periods of $1.5 \mathrm{sec}$ in intervals of $9.3 \mathrm{sec}$. The size of the gratings was such that the HS- and FD-cells in the visual systems of the animals were stimulated about equally strongly (see Hausen and Wehrhahn, 1989).

The flies were fixed to a torque meter and were adjusted with respect to the stimulus under microscopic control. Their frontal equatorial pseudopupils, which indicate position and orientation of the heads, were used as optical cues for the adjustment (Wehrhahn, 1978). The stimulusinduced yaw torque responses of the tethered flying animals were recorded, digitized, and stored for later averaging. The experimental setup is sketched in Figure $1 a$.

In a first series of tests on 5 flies, the effects of the control operation were studied by comparing the torque responses recorded before and after the operation. Consistent behavioral changes that could be attributed to this operation were not observed.

In a second series of pilot experiments performed with 4 operated flies (lesion $\mathrm{L} 1$ ), the responses to monocular and binocular stimulation with horizontal motion were measured over $4 \mathrm{~d}$ after the operation. With these measurements we investigated whether slow behavioral alterations, e.g., due to progressive lesion-induced neural degeneration, or perhaps even regeneration occurred in this period. Although consistent changes of the responses were not observed, behavioral tests of lesioned animals were exclusively performed on the first day after operation in the final experiments.

\section{Histological controls}

After the behavioral tests the brain of each operated animal was immediately fixed in AAF, embedded in paraffin, cut in $10-\mu \mathrm{m}$ frontal serial sections, and stained with Toluidine blue. The sections were examined in order to check the position and size of the lesion in the neuropil. Behavioral data from animals showing incorrect lesions were omitted from further evaluation.

\section{Results}

Yaw torque responses to horizontal grating motion

Responses of normal flies. In initial experiments, the transient yaw torque responses of normal, unoperated blowflies and houseflies to monocular (Fig. $2 a$ ) and binocular (Fig. $2 b$ ) horizontal motion were recorded. The results (Fig. 2, upper traces) demonstrate that in both species of flies monocular motion from front to back and from back to front and binocular rotatory motion elicit strong optomotor torque responses, whereas monocular motion from back to front is a significantly less effective stimulus. The responses to binocular translatory motions (binocular motion from front to back and from back to front) are zero because of symmetry. This is in full agreement with the results of the detailed interspecific comparison performed in the first part of this study and supports the view again that both species have very similar visual torque control systems. Two minor differences will be mentioned briefly: the response decay after stimulation with monocular motion from front to back and binocular rotatory motion is slower in $\mathrm{Musca}$ than in $\mathrm{Cal}$ liphora, and the optomotor torque response to monocular motion from back to front is observed only in Musca. As will be discussed below, these differences can readily be explained within the framework of the proposed cellular circuitry and do not indicate different cellular wirings of the torque control systems in the 2 species.

Responses of operated flies. The results of the lesion experiments, performed on Calliphora are compiled in the lower part of Figure 2, which shows the response traces of animals with lesion $\mathrm{L} 1$ and of animals with lesion $\mathrm{L} 2$.

We will first consider the responses marked 1-6 and use the diagrams of Figure 3 for discussion. Diagram 1 in Figure $3 a$ illustrates that, in normal flies, the response to monocular motion from front to back in the right visual field is induced by the FD4 and the HS-cells of the right lobula plate. Both cells induce positive torque responses, i.e., optomotor turns of the fly to the right side. In animals with lesion L1 (2) the torque component controlled by the HS-cells is expected to vanish, the remaining torque signal reflecting solely the activity of the FD4. In contrast, the torque responses of animals with lesion L2 (3) should be solely controlled by the HS-system. Hence, the qualitative effect to be expected in these experiments is a reduction of the torque amplitude in animals with $\mathrm{L} 1$ and $\mathrm{L} 2$ as compared to the response of normal animals. The data in Figure $2 a \mathrm{dem}$ onstrate that this is indeed the case.

Under binocular stimulation with clockwise rotatory motion (4-6), the left eye is stimulated additionally with motion from back to front. As is illustrated in Figure $3 b$, the response of the normal animal is composed of positive and negative components under this condition. The right HS-system is now additionally activated by the $\mathrm{H} 2$ of the left lobula plate and should induce an increased positive torque component. In addition, the left FD3 and the right FD4 are activated, which should lead to positive and negative torque response components, respectively. However, since stimulation is binocular, both FD-cells receive strong inhibitory influences. The effect of this inhibition can readily be observed when comparing responses 2 and 5 in Figure 2. Under both monocular and binocular stimulation, the measured response is assumed to be solely controlled by the FD4, since all other activated cells are lesioned (it is assumed here that the inactivated cells are in a state that does not influence the FD4). Whereas under monocular stimulation of the right eye a significant positive response is elicited (2), that response vanishes completely when the left eye is additionally stimulated with motion in the same direction (5), which in normal animals elicits a strong optomotor torque response (4). We thus conclude that extinction of the response in 5 may be due to complete inactivation of the FD4 under binocular stimulation.

A similar consideration leads to the inference that binocular motion in the opposite direction leads to full inactivation of the FD3. Hence, the response of the intact animal to binocular rotation (4) should be induced only by the H2- and HS-cells, whereas in animals with lesion L2 (6) only the right HS-cells control torque generation. A comparison of the data of Figure 2 shows that response 6 is indeed smaller than response 4 . Furthermore, the responses of animals with lesion L2 to monocular and binocular stimulation are about equal in amplitude, as expected (compare 3 and 6 in Figs. 2 and 3). Critical examination of the data obtained under the other combinations of lesions and stimulation conditions compiled in Figure 2 reveals similar qualitative agreements between measured and predicted responses.

In order to perform a more precise comparison between experimental data and predictions, one can extract the individual response components quantitatively from those experiments where, according to our concept, l cell solely determines the response of the animal. Under the simple assumption that all components add linearly, the respective contributions of all cells can be determined. These values, which are compiled in Table 1 , can then be used to make quantitative predictions about the responses of normal and lesioned animals. These calculated responses are shown as arrows in Figure 2, indicating the expected polarity and amplitude of the torque responses elicited under the various experimental conditions. A comparison demonstrates that measured and predicted responses are, in general, 


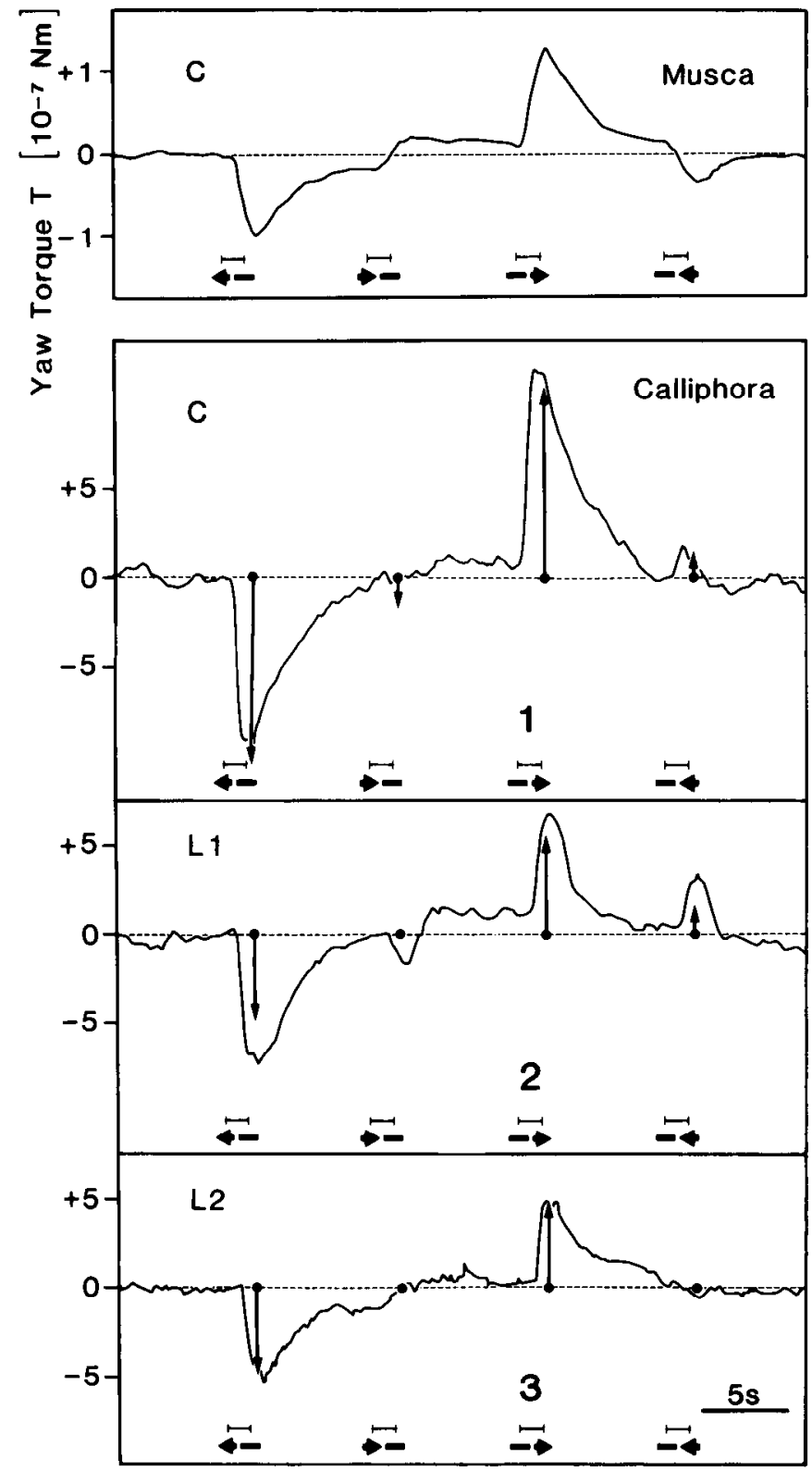

Time
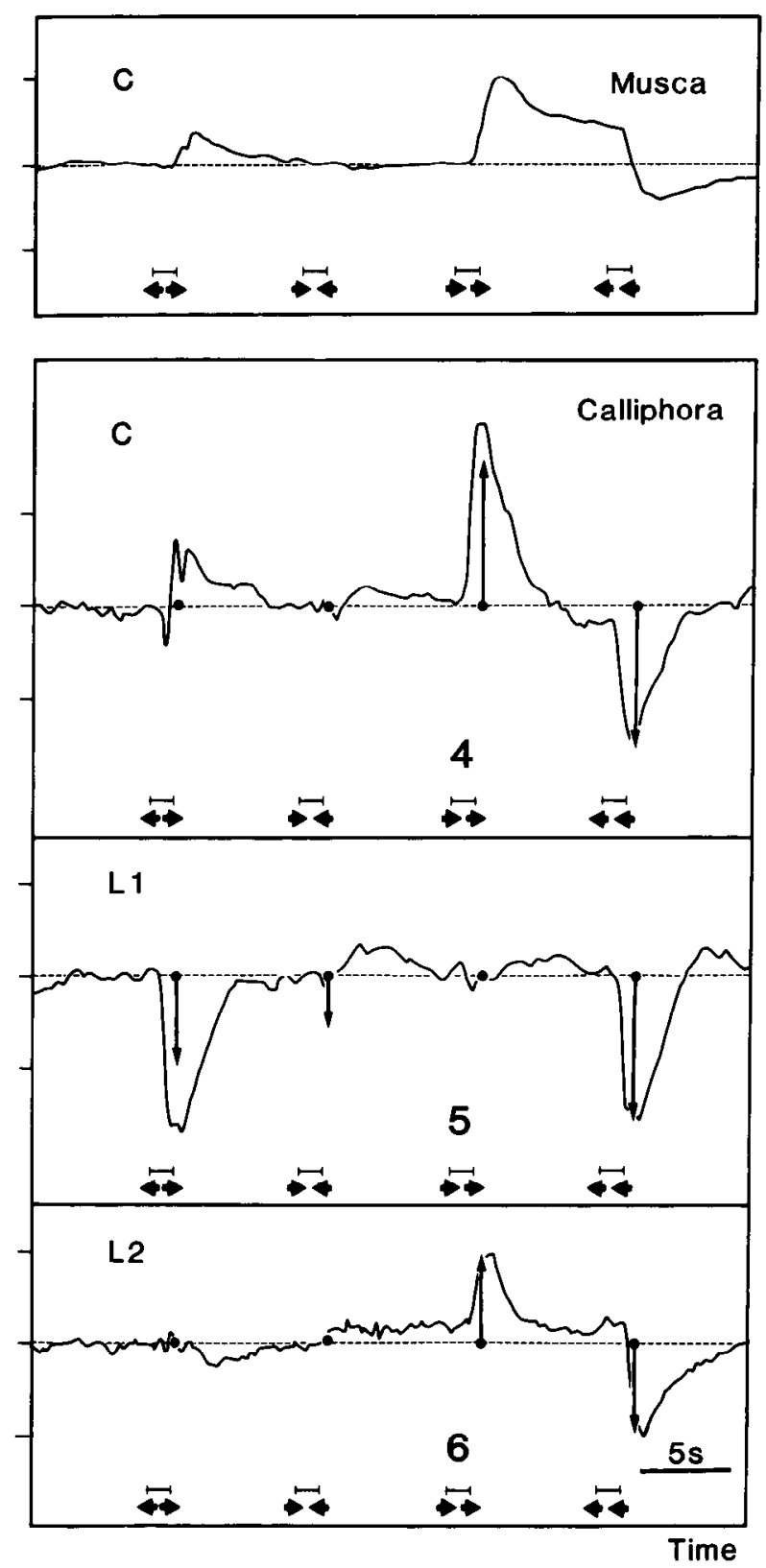

a

b

Figure 2. Yaw torque responses of normal control fies $(C)$ and operated flies with lesion $L 1$ and $L 2$ to $(a)$ monocular and $(b)$ binocular stimulation with horizontal grating motion. Control measurements $(C)$ were performed on Musca and Calliphora; measurements on lesioned animals were performed only on Calliphora. The torque responses were recorded under the experimental condition illustrated in Figure 1a. The individual motion stimuli are indicated below the response traces. The traces shown represent normalized response averages obtained from $250-530$ stimulation sequences in 10-33 flies. Positive and negative torque values represent intended turns in clockwise and counterclockwise direction. Arrows indicate amplitudes and polarities of expected yaw torque responses calculated on the basis of the proposed concept of the yaw torque control system outlined in Figure 1c. The traces demonstrate that monocular motion from front to back and binocular rotatory motion induce strong optomotor yaw torque responses in both species of flies. The brain lesions lead to characteristic impairments of the response which are discussed in detail in the text. The composition of the responses under experimental conditions $1-6$ is illustrated in Figure 3 .

in good agreement with each other. Since the predictions are made on the basis of the wiring model that is highly specific with respect to the functional and anatomical properties of the individual cells, we regard this close agreement as convincing evidence that the proposed model is a sufficient representation of the torque control system in the brain of the fly.

\section{Yaw torque responses to vertical grating motion}

In a second series of experiments the yaw torque responses to monocular grating motion in vertical direction were investigated in normal and operated blowflies. The results of these measurements are shown in Figure 4. 
Figure 3. Expected contributions of lobula plate output neurons to torque generation under monocular and binocular stimulation with motion in normal and lesioned animals. In the individual wiring diagrams, the cells activated by the motion stimulus are drawn in heavy lines, and the cells expected to contribute to the torque responses are listed at the bottom. Diagrams $1-6$ refer to the experimental conditions marked $1-6$ in Figure 2. The figure illustrates that the motor output (torque $\mathrm{T}$ ) of normal flies is composed of different components which can be partially eliminated by the different combinations of lesions and stimulation conditions. See text for further explanation.
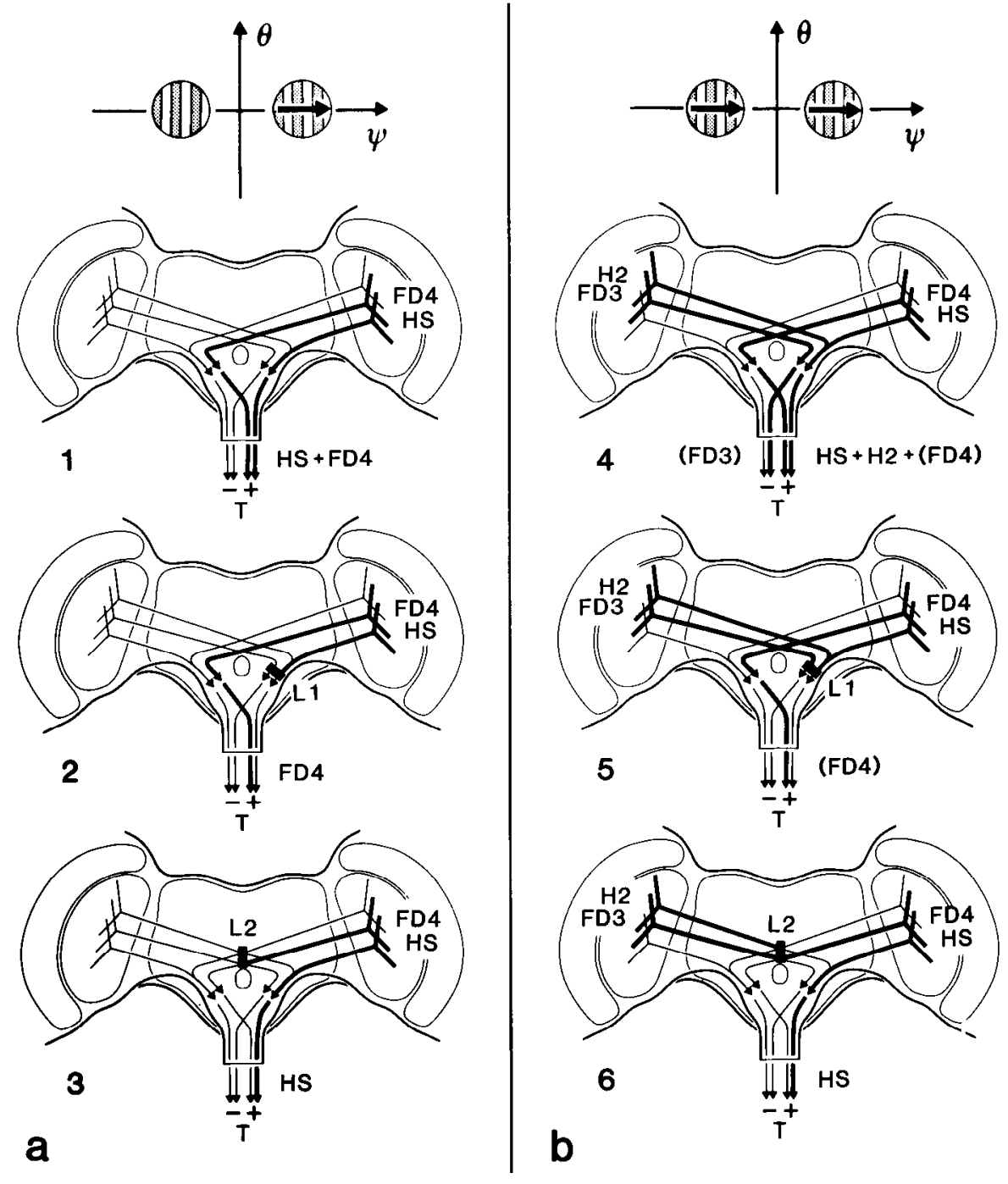

The normal blowflies show significant torque responses to vertical motion. In contrast to the responses to horizontal motion, the responses elicited in this case are directed toward the stimulus irrespective of the direction of motion. This excludes the possibility that these responses are due to horizontal motion components seen by the animal because of misalignment between the preferred directions of the movement detectors in the stimulated eye region and the orientation of the grating. In this case, responses of opposite polarity would be elicited by motion in opposite directions. The torque responses of operated flies (lesion L1) recorded under these stimulus conditions remained more or less unaffected by the lesion.
At present, the cellular elements controlling these responses are not identified. The lesion experiments indicate, however, that the axonal pathways of these cells do not transverse the operated region of the deutocerebrum.

\section{Discussion}

Neural control of yaw torque in flies: experimental findings and inferences

In the strict sense, particular neural circuits can only be shown to control a particular stimulus-induced behavior by analyzing anatomically and physiologically all elements of the neural pathway between sensory input and motor output. Concerning the

Table 1. Yaw torque components induced by individual cells of the lobula plate

\begin{tabular}{lllll} 
Cell direction & $\begin{array}{l}\text { HS } \\
\text { front/back }\end{array}$ & $\begin{array}{l}\text { H2 } \\
\text { back/front }\end{array}$ & $\begin{array}{l}\text { FD4 } \\
\text { front/back }\end{array}$ & $\begin{array}{l}\text { FD3 } \\
\text { back/front }\end{array}$ \\
\hline Induced torque $\left[10^{-7} \mathrm{~nm}\right]$ & +4.9 & -2.9 & +5.6 & +4.5
\end{tabular}

For sign of responses note projection side of individual elements leaving the cervical connective (see wiring diagrams of Fig. 3). The contributions from the FD cells under binocular stimulation are considered to be negligible and hence set to zero. The data were obtained from the measurements shown in Figure 2 (values in $10^{-7} \mathrm{~nm}$ ). 
neural circuity controlling the motion-induced yaw torque responses in flies, we are confronted with 2 major problems. First, the bulk of the behavioral data available was obtained from Musca and Drosophila, whereas all electrophysiological and most anatomical data relevant in this context were obtained from Calliphora. Second, we know the functional and structural properties of the interneurons of the lobula plate rather well and have some information about the motor system, but little is known about the linking descending neurons in the cervical connective.

For these reasons the first goal of the 2 present studies was to compare functional characteristics of the torque control systems in Calliphora and Musca. This was done mainly in the first part of this study by comparing the transient yaw torque responses of both species to horizontal and vertical grating motion. The results demonstrate that the torque responses in both species are, in general, very similar. The present data on the transient responses of both species support this view. Minor differences between Musca and Calliphora, as revealed in Figure 2 , concern the response decays after stimulation with front-toback motion and the responses to regressive motion. The former may be explained by slightly different filter characteristics in the pathway between lobula plate and motor system, whereas the latter may be due to a different weighting of the opposing effects of the FD-cells and the H2-cells on torque generation in the 2 species. Neither possibility implies strongly different neural mechanisms. This conclusion is corroborated by a similar comparative investigation of the yaw torque responses of Calliphora and Musca performed under different stimulation conditions (Egelhaaf, 1985a, c) and agrees well with basic similarities found in a number of other studies, including extensive work on Drosophila, which have already been discussed in the preceding paper. We thus infer that the neural torque control systems in all 3 species are very similar.

The second goal of our study was to test the validity of the proposed torque control circuitry, without analyzing the entire pathway between lobula plate and motor system. This was done in 2 steps. In the first step the functional characteristics of the HS-cells and the torque control system were evaluated in a combined electrophysiological and behavioral study. The compatibility of the obtained physiological and behavioral data is prerequisite for the validity of the proposed wiring concept but by no means excludes alternative neural control circuits. In a similar approach, the responses of HS- and FD-cells to oscillating motion patterns modeled in computer simulations were used to simulate the behavioral responses of the fly (Egelhaaf, 1985 c). Although even highly specific behavioral response transients could be simulated satisfactorily in this way, this approach yielded evidence in favor of, but not proof of, the concept. In contrast to these approaches, the lesion experiments performed here represent a rigid and very direct test of the proposed torque control circuitry, and we regard the results as convincing evidence for its correctness.

\section{The reliability of brain lesions as experimental tools}

Conclusions about wiring and functional significance of neural circuits in the brain that are drawn from lesion-induced behavioral changes must be treated with caution for several reasons. First and most important, one cannot exclude the possibility that apart from the intended neurons other cells were impaired which contribute significantly to the behavior studied. This is particularly so when lesions are performed in dense neuropils

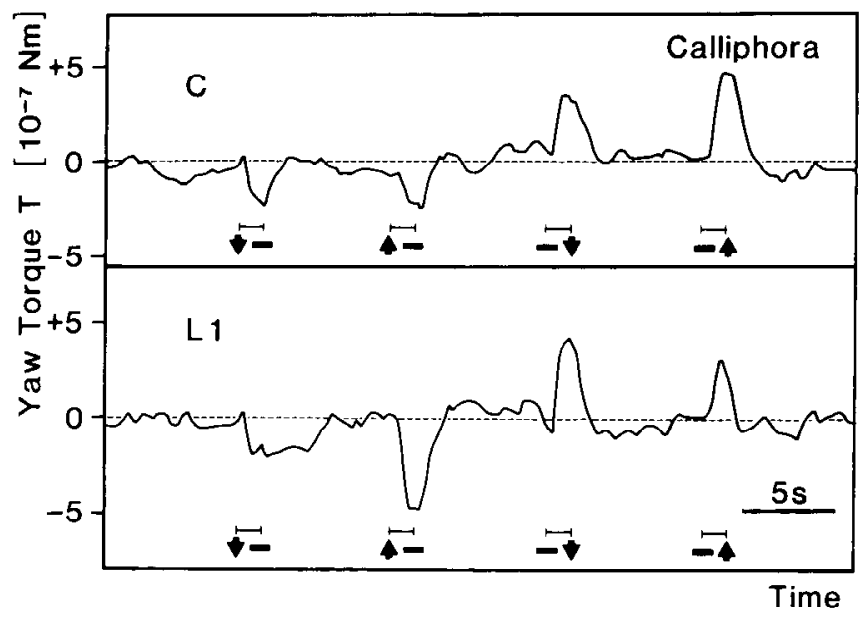

Figure 4. Yaw torque responses of normal control flies $(C)$ and operated flies with lesion $L l$ to stimulation with vertical motion presented in the right or left visual field. Control experiments and lesion experiments were exclusively performed with Calliphora. Except for the direction of motion, the experimental conditions were identical to those of Figure 2. The individual motion stimuli are indicated below the response traces. The traces represent normalized averages obtained from measurements under 390-450 stimulation sequences in 10-28 flies. The torque responses under the present conditions are directed toward the moving grating irrespective of the direction of motion. The responses in Calliphora are not impaired by lesion $\mathrm{L} 1$.

as is the case in the present experiments. Second, the entire operation (e.g., opening of head capsula, etc.) and subsequent neural degenerations may have unspecific effects on the behavior investigated. We therefore performed rigid controls.

In pilot experiments the responses of lesioned animals were first examined individually in order to find out whether consistent behavioral changes could be observed at all. This turned out to be the case. Subsequently the behavioral responses of animals treated with all steps of the operations except for the actual brain lesions were measured. We thereby investigated whether the bchavioral changes obscrved in the opcrated animals were in fact due to the lesions or only to the operational procedure. These tests demonstrated that the responses of animals treated with such control operations remained normal. In order to exclude further that the data obtained were influenced by slow behavioral degradations due to progressive neural degeneration, the behavioral stability of lesioned animals was tested in initial experiments during $3 \mathrm{~d}$ after operation. Although indications of consistent behavioral changes during this period were not observed, all data presented were obtained exclusively on the first day after operation. Finally, all brains were histologically examined in order to control the correct position of the lesions before including the respective behavioral data in the final response averages.

In spite of these controls we are fully aware of the possibility that in all lesions more than the intended neurons were impaired. We regard the present data, nevertheless, as significant since the behavioral changes caused by the operations are highly specific and can be consistently interpreted in terms of a rather complex neural circuitry. This consistency can hardly be accidental nor does it seem likely that the same consistency between experimental data and predictions can be derived simply from an alternative circuitry with different structural and functional properties. 


\section{Experimental data of this study and predictions: a critical discussion}

The yaw torque responses of normal and lesioned flies evaluated under the different stimulus conditions of the present experiments agree qualitatively very well with the predictions derived from the proposed circuitry. Furthermore, there is, in general, even a rather good quantitative agreement between measured and predicted responses, as shown in Figure 2. Minor discrepancies occur mainly in the data on lesion L1 and may be explained by incomplete elimination of the terminals of the left FD-cells by this lesion. This may occasionally have happened, since the FD-terminals consist of a number of thin profiles which could not be resolved in the histological controls performed after the behavioral tests. Therefore, we concentrated in these controls on the prominent HS-axons, which could always be identified in the sections and in which lesions could unequivocally be determined. Because the FD-terminals are closely opposed to the axonal endings of the HS-cells, a visible lesion of the latter was taken as evidence that the FD-terminals were also cut. A recalculation of the predicted responses of animals with lesion L1 under the worst assumption, namely, that the FDterminals remained completely unimpaired and that only the tight HS-system and the synaptically linked terminal of the left $\mathrm{H} 2$ were cut in this operation, yields changes in the predicted response amplitudes; e.g., the predicted responses to monocular stimulation with horizontal motion in the left visual field are now identical to those of the unoperated control animals, whereas the predicted responses to monocular stimulation in the right visual field remain unaltered. A comparison with the measured responses reveals that their amplitudes reach intermediate values between the 2 predictions (see Fig. 2). The situation is similar when recalculating the responses to binocular stimulation in animals with lesion L1 (although the comparison may not be valid in the strict sense for the binocular stimulus condition). This consideration indicates that these quantitative discrepancies between measurement and interpretation can be explained within the framework of the proposed circuitry by an incomplete elimination of the FD-terminals and does not lead to different inferences concerning the structural and functional organization of the neural torque control system.

A further point to be discussed regards the inhibition of the FD-cells under binocular stimulation with motion. We concluded from our data that the FD-cells are fully inhibited under this condition. At present, one cannot substantiate this inference by independent electrophysiological data, since the functional properties of the FD-cells have so far been studied only under rather different stimulation conditions (Egelhaaf, 1985b). Our data indicate, however, that under the present binocular stimulation conditions the FD-cells should be silent.

Finally we would like to discuss the fact that we did not include the cells FD1 $1_{\text {pos }}$ and FD2 into the quantitative considerations outlined above (see also Cellular Organization of the Two Control Circuits).

The receptive fields of $F D 1_{\text {pos }}$ and FD2 are situated in the frontal region of the compound eyes. They are, therefore, stimulated only weakly in our experiments. In addition, there is no response to stimulation with motion from back to front. Since FD2 is sensitive to motion in this direction, it is unlikely to contribute to this yaw torque response.

Comparison of the experiments with monocular progressive and binocular clockwise rotatory motion when lesion L2 was applied shows that the amplitudes of all responses are roughly equal. We conclude from this that these responses are controlled only by the HS and that in particular $\mathrm{FDI}_{\text {pos }}$ does not contribute.

We cannot, however, exclude the possibility that $\mathrm{FDl}_{\text {nod }}$ contributes to the observed responses. Therefore the components described as driven by FD4 could in addition be driven by $F D 1_{\text {nod }}$. This would imply no principal change in the circuitry outlined.

\section{Is there an additional torque control system tuned to local vertical motion?}

The experiments shown in Figure 4 demonstrate that yaw torque responses toward the stimulus can be elicited by monocular vertical motions in both directions. Thinking in terms of classical optomotor torque responses to large-field stimulation, these responses can hardly be understood. Flies are well known to generate torque responses about the longitudinal body axis under these stimulations (roll responses) which, however, were not measured in the present experiments. Even if the recorded yaw responses are by-products of intended roll movements of the animals, as can be observed in banked turns during free flight (Wagner and Wehrhahn, 1985; Wagner, 1986), one would not expect responses of the same polarity to both directions of motion. The latter argument also excludes the possibility that the animals responded to horizontal motion components resulting from misalignments of the gratings.

The results can be understood, however, if one assumes a second system tuned to local motion in the visual system which responds selectively to motion in the vertical direction. In analogy to the known FD-cells, such a system should induce turnings toward the moving stimulus irrespective of the direction of motion. It is tempting to assume its existence, since it would considerably improve the capability of the fly to detect and fixate objects. This system would show functional properties at the yaw torque output very similar to the position coding system described earlier (Pick, 1974; Reichardt and Poggio, 1976; Reichardt, 1979; Bülthoff and Wehrhahn, 1984).

\section{The lobula plate: a center for motion computation for $3 D$ course control}

The neural circuitry investigated here is only a small part of a complex motion computation network in the lobula plate of the fly which seems to control various motor outputs involved in the 3D course control in flight. This network has been thoroughly analyzed in recent years (Hengstenberg, 1984; Egelhaaf et al., 1988; Hausen and Egelhaaf, 1989), and at present about 50 large interneurons have been identified, all of which are directionally selective motion-sensitive interneurons. Physiologically the individual cells differ from each other mainly with regard to their receptive field organizations, preferred directions, and spatial integration properties. Concerning the role in course control, one can subdivide the entire array into circuits controlling yaw, roll, and pitch responses. At least the yaw torque control system is further divided into subcircuits selectively processing global and local retinal motion patterns. Although only the HS-cells and the cells FD1-4 have been considered so far, a number of further cells of the lobula plate are very likely to be involved in these computations. These are the elements presumably mediating the binocular inhibition of the FD-cells under large-field stimulation (e.g., the CH-cells, $\mathrm{Hl}$-cells, and H5-cells; see Hausen, 1989). In addition, further cells scanning the posterior part of the visual field and responding selectively 
to progressive and regressive motion (H3-, H4-cells), which belong to the same anatomical class as the heterolateral FDcells discussed here, may eventually turn out to be also FDcells. In this case, the entire visual field of the fly would be scanned by FD-cells.

The major output cells coding self-rotation about the longitudinal and transverse body axis (roll and pitch) are the wellknown giant vertical cells (Hengstenberg, 1984). Whether an FD-system sensitive to vertical motion also exists in the lobula plate remains to be investigated. The present results (Fig. 4) may indicate such a system, and potential cellular candidates of it exist in the lobula plate (vCal 1-3). In addition, various cells sensitive to vertical motion and projecting into the contralateral lobula plate may well be involved in the binocular inhibitory processes underlying the tuning to local motion sensitivity. A thorough investigation of the functional role of the VS-cells in roll and pitch control equivalent to the present study has not yet been performed. In analogy to the HS-system, the significance of the VS-system seems, however, evident.

Thus, the yaw torque control circuits investigated here are part of a complex 3D course control system in the lobula plate, which monitors the global and local motion patterns perceived by the compound eye and governs the steering maneuvers in flight. There is evidence that further components of motioninduced behavioral responses, such as head movements (Hengstenberg, 1984; Milde and Strausfeld, 1986) and rudder movements of the abdomen and hindlegs (Götz, 1968; Zanker, 1988), are also controlled by the motion computation circuits in this neuropil.

\section{References}

Bülthoff, H., and C. Wehrhahn (1984) Computation of motion and position in the visual system of the fly $(M u s c a)$. Experiments with uniform stimulation. In Localization and Orientation in Biology and Engineering, Varju and Schnitzler, eds., Springer-Verlag, Berlin.

Eckert, H. (1981) The horizontal cells in the lobula plate of the blowfly, Phaenicia sericata. J. Comp. Physiol. 143: 511-526.

Egelhaaf, M. (1985a) On the neuronal basis of figure ground discrimination by relative motion in the visual system of the fly. I. Behavioural constraints imposed on the neuronal network and the role of the optomotor system. Biol. Cybern. 52: 123-140.

Egelhaaf, M. (1985b) On the neuronal basis of figure ground discrimination by relative motion in the visual system of the fly. II. Figuredetection cells, a new class of visual interneurones. Biol. Cybern. 52: 195-208.

Egelhaaf, M. (1985c) On the neuronal basis of figure ground discrimination by relative motion in the visual system of the fly. III. Possible input circuitries and behavioural significance of the FD-cells. Biol. Cybern. 52: 267-280.

Egelhaaf, M. (1989) Visual afferences to flight steering muscles controlling optomotor responses of the fly. J. Comp. Physiol. (in press).

Egelhaaf, M., K. Hausen, W. Reichardt, and C. Wehrhahn (1988) Visual course control of flies relies on neuronal computation of object and background motion. TINS 11:351-358.

Fermi, G., and W. Reichardt (1963) Optomotorische Reaktionen der Fliege Musca domestica. Kybernetik 2: 14-28.

Götz, K. G. (1968) Flight control in Drosophila by visual perception of motion. Kybernetik 4: 199-208.

Götz, K. G. (1983) Bewegungssehen und Flugsteuerung bei der Fliege Drosophila. In Biona-Report 2, W. Nachtigall, ed., pp. 21-23, Gustav Fischer, New York.

Hausen, K. (1976) Functional characterization and anatomical identification of motion sensitive neurons in the lobula plate of the blowfly Calliphora erythrocephala. Z. Naturforsch. 31c: 629-633.

Hausen, K. (1981) Monocular and binocular computation of motion in the lobula plate of the fly. Verhandl. Deutsch. Zool. Ges. 1981: $49-70$.

Hausen, K. (1982a) Motion sensitive interneurons in the optomotor system of the fly. I. The horizontal cells: Structure and signals. Biol. Cybern. 45: 143-156.

Hausen, K. (1982b) Motion sensitive interneurons in the optomotor system of the fly. II. The horizontal cells: Receptive field organization and response characteristics. Biol. Cybern. 46: 67-79.

Hausen, K. (1984) The lobula-complex of the fly: Structure, function and significance in visual behaviour. In Photoreception and Vision in Invertebrates, M. A. Ali, ed., pp. 523-559, Plenum, New York.

Hausen, K. (1989) The neural architecture of the lobula plate of the blowfly, Calliphora erythrocephala. Cell Tissue Res. (in press).

Hausen, K., and M. Egelhaaf (1989) Neural mechanisms of visual course control in insects. In Facets of Vision, D. Stavenga and R. Hardie, eds., Springer-Verlag, Berlin, Heidelberg, New York (in press).

Hausen, K., and R. Hengstenberg (1987) Multimodal convergence of sensory pathways on motoneurons of flight muscles in the fly ( $\mathrm{Cal}$ liphora). Soc. Neurosci. Abstr. 13: 1059.

Hausen, K., and C. Wehrhahn (1983) Microsurgical lesion of horizontal cells changes optomotor yaw responses in the blowfly Calliphora erythrocephala. Proc. R. Soc. Lond. B 219: 211-216.

Hausen, K., and C. Wehrhahn (1989) Neural circuits mediating visual flight control in flies. I. Quantitative comparison of neural and behavioral response characteristics. J. Neurosci. 9: 3828-3836.

Hausen, K., K. Wolburg-Buchholz, and W. Ribi (1981) The synaptic organization of visual interneurones in the lobula complex of flies. A light and electron microscopical study using silver-intensified cobalt impregnations. Cell Tissue Res. 208: 371-387.

Hausen, K., R. Hengstenberg, and T. Wiegand (1988) Flight control circuits in the nervous system of the fly: Convergence of signal and mechanosensory pathways onto motoneurons of steering muscles. In Sense Organs, N. Elsner and F. G. Barth, eds., Proceedings of the 16th Göttingen Neurobiology Conference, p. 130, G. Thieme Verlag, Stuttgart.

Heide, G. (1975) Properties of a motor output system involved in the optomotor response in flies. Biol. Cybern. 20: 99-112.

Heide, G. (1983) Neural mechanisms of flight control in diptera. In Biona-Report 2, W. Nachtigall, ed., pp. 35-52, Gustav Fischer, New York.

Heisenberg, M., and R. Wolf (1984) Vision in Drosophila, SpringerVerlag, New York.

Heisenberg, M., R. Wonneberger, and R. Wolf (1978) Optomotorblind $^{\mathrm{H} 31}$ - A Drosophila mutant of the lobula plate giant neurons. J. Comp. Physiol. 124: 287-296.

Hengstenberg, R. (1984) Roll-stabilization during flight of the blowflics head and body by mechanical and visual cucs. In Localization and Orientation in Biology and Engineering, Varju and Schnitzler, eds., pp. 121-134, Springer-Verlag, Berlin.

Hengstenberg, R., K. Hausen, and B. Hengstenberg (1988) Cobalt pathways from haltere mechanoreceptors to inter- and motoneurons controlling head posture and flight steering in the blowfly Calliphora. In Proceedings of the 16th Göttingen Neurobiology Conference, $\mathrm{N}$. Elsner and F. G. Barth, eds., p. 129, Thieme Verlag, Stuttgart.

Hirth, C. (1981) Elektrophysiologische Untersuchungen über die Bildung der Impulsmuster in den neuromotorischen Systemen nicht-fibrillärer Flugmuskeln von Schmeissfliegen (Calliphora). Doctoral Dissertation, Universität Düsseldorf.

Milde, J. J., and N. J. Strausfeld (1986) Visuomotor pathways in arthropods. Naturwissenschaften 73: 151-165.

Pick, B. (1974) Visual flicker induces oricntation behaviour in the fly Musca. Z. Naturforsch. 29c: 310-312.

Pick, B. (1976) Visual pattern discrimination as an element of the fly's orientation behaviour. Biol. Cybern. 23: 171-180.

Poggio, T., W. Reichardt, and K. Hausen (1981) A neuronal circuitry for relative movement discrimination by the visual system of the fly. Naturwissenschaften 68: 443-446.

Reichardt, W. (1965) Quantum sensitivity of light receptors in the compound eye of the fly Musca. In Cold Spring Harbour Symposia on Quantitative Biology 1965, pp. 505-51 5, Cold Spring Harbor Press, Cold Spring Harbor, NY.

Reichardt, W. (1973) Musterinduzierte Flugorientierung. VerhaltensVersuche an der Fliege Musca domestica. Naturwissenschaften 60 . 122-138.

Reichardt, W. (1979) Functional charactcrization of ncural intcraction through an analysis of behaviour. In The Neurosciences, Fourth Study Program, F. O. Schmidt and F. G. Worden, eds., pp. 81-103, MIT Press, Cambridge, MA. 
Reichardt, W. (1986) Processing of optical information by the visual system of the fly. Vision Res. 26: 113-126.

Reichardt, W., and T. Poggio (1976) Visual control of orientation behaviour in the fly. Part I. A quantitative analysis. Q. Rev. Biophys. 9: 311-375.

Reichardt, W., T. Poggio, and K. Hausen (1983) Figure-ground discrimination by relative movement in the visual system of the fly. Part II: Towards the neural circuitry. Biol. Cybern. 46(Suppl.): 1-30.

Spüler, M. (1980) Erregende und hemmende Wirkungen visueller Bewegungsreize auf das Flugsteuersystem von Fliegen-Elektrophysiologische und verhaltensphysiologische Untersuchungen an Musca und Calliphora. Doctoral Dissertation, Universität Düsseldorf.

Spüler, M., and G. Heide (1987) Simultaneous recording of torque, thrust and muscle spikes from the fly Musca domestica during optomotor responses. Z. Naturforsch. 33c: 455-457.

Strausfeld, N. J., and J. Bacon (1983) Multimodal convergence in the central nervous system of dipterous insects. In Fortschritte der Zoologie, Bd. 28: Horn (hrsg.), Multimodal Convergence in Sensory Systems, Gustav Fischer Verlag, New York.

Strausfeld, N. J., and U. K. Bassemir (1985) The organization of giant horizontal-motion-sensitive neurons and their synaptic relationships in the lateral deutocerebrum of Calliphora erythrocephala and Musca domestica. Cell Tissue Res. 242: 531-550.
Wagner, H. (1986) Flight performance and visual control of flight of the free-flying housefly (Musca domestica L.). I. Organization of the flight motor. Phil. Trans. R. Soc. Lond. [Biol.] 312: 527-551.

Wagner, H., and C. Wehrhahn (1985) Head-body coordination in free flying houseflies: Aerodynamic performance and visual flow. Soc. Neurosci. Abstr. 11: 498.

Wehrhahn, C. (1978) Flight torque and lift responses of the housefly (Musca domestica) to a single stripe moving in different parts of the visual field. Biol. Cybern. 29: 237-247.

Wehrhahn, C. (1985) Visual guidance of flies during flight. In Comprehensive Insect Physiology, Biochemistry and Pharmacology, Vol. 6, Nervous Systems: Sensory, G. A. Kerkut and L. I. Gilbert, eds., pp. 673-684, Pergamon, Oxford.

Wehrhahn, C. (1986) Motion sensitive torque responses of the housefly Musca: A quantitative study. Biol. Cybern. 55: 275-280.

Wehrhahn, C., and K. Hausen (1980) How is tracking and fixation accomplished in the nervous system of the fly" A behavioural analysis based on short time stimulation. Biol. Cybern. 38: 179-186.

Zanker, J. M. (1988) How does lateral abdomen deflection contribute to flight control of Drosophila melanogaster? J. Comp. Physiol. 162: 518-528. 